$\ll$ Research Note»

\title{
Effect of Dwarfing Gene dw on Erythrocyte Counts, Hematocrits and Hemoglobin Concentrations in Chickens during 1 to 30 Weeks of Age
}

\author{
Sadao Hoshino, Issei Y амамото and Masaaki Wakita \\ Faculty of Bioresources, Mie University, Tsu 514
}

\begin{abstract}
Some hematological differences between dwarf (Gifu 20 line) and normal (Gifu 15 line) chickens were studied during the period of growth from 1 to 30 weeks of age. Erythrocyte counts, hematocrits and hemoglobin concentrations were mostly significantly higher for dwarf than for normal chickens. This paradoxical increase appeared to be related with hormonal status in the dwarf chicken.
\end{abstract}

(Jpn. Poult. Sci., 29 : 151-155, 1992)

Key words : dwarf, growth, erythrocyte, hematocrit, hemoglobin,

\section{Introduction}

The recessive sex-linked dwarf chicken is characterized by reduced body weight and shortened shank length together with lower plasma levels of insulin-like growth factor (IGF)-1 and triiodothyronine than in the normal chicken ${ }^{1)}$. Recently it becomes clearer that IGF-1 as well as growth hormone (GH) stimulates both erythropoiesis and Fe incorporation into reticulocytes and thereby increases blood erythrocyte numbers ${ }^{2}$, and that there is a linear correlation between blood IGF- 1 concentrations and daily red blood cell formation in growing rats $^{3)}$. Considering a low circulating IGF-1 level in the dwarf chicken ${ }^{4,5)}$, it appeared to be of interest to investigate whether the dwarfing gene exerts its influence on red blood cell numbers and hemoglobin $(\mathrm{Hb})$ contents.

Blood constituents of dwarf birds have so far been compared with normal birds, but the comparison is made only in two age groups: young and adult chickens ${ }^{6,7)}$. Thus we report here some blood parameters of dwarf and normal cockerels and pullets during their growth period from hatching to adult.

\section{Materials and Methods}

Dwarf chicks were obtained from a stock of dwarf chicken (Gifu 20 line) maintained in the Gifu Poultry Breeding Station. The dwarf line was made by repeated backcrosses of Dwdw males (at first M-strain, White Leghorn) to Dw females (15 line, Rhode Island Red $)^{8}$. The control normal chicks were obtained from the 15 line of this station. Although the 15 line is strictly not a proper control, it has more genes in common with the 20 line than any other strains readily available. 
Chicks were housed in an electric brooder for 4 weeks and thereafter in an unheated brooder. The birds were fed a commercial diet and water ad libitum.

Body weights and shank lengths (shank-toe lengths) were measured at 1, 3, 5, 7, 9, $11,13,15,17,19,21,23,25$ and 30 weeks of age. Blood samples were taken with a heparinized syringe either by cardiac puncture or venipuncture at the same days of body weight measurement and the following hematological parameters were obtained : erythrocyte count, hematocrit and $\mathrm{Hb}$ content. Erythrocytes were counted in a Thoma-typehemocytometer. Hematocrits were determined with the aid of Wintrobe tubes and centrifugation (3000 r.p.m., 30 min.). Hb contents were estimated spectrophotometrically $(540 \mathrm{~nm})$ as cyanmethemoglobin. The data were examined for statistical differences by Students $t$ test.

\section{Results and Discussion}

In cockerels, body weights of dwarf chickens were significantly reduced during 7 to 30 weeks of age, while in pullets the reduction was not significant up to 13 weeks of age (Table 1). In contrast, the depressing effect of dw gene on skeletal growth was evident at earlier weeks of growth (Table 2). There were significant differences in body weights and shank lengths between male and female chickens of each line (Tables 1 and 2). These results are consistent with those reported partially for these normal and dwarf lines ${ }^{8,9)}$.

Changes in erythrocyte count, hematocrit and $\mathrm{Hb}$ content during growth of chickens are shown in Tables 3-5, respectively. Erythrocyte numbers were rather lower in dwarf male chickens than in normals until 5 weeks of age, but after 7 weeks of age they were consistently higher in dwarf males with significant differences.

Table 1. Body weights $(\mathrm{g})($ Mean $\pm \mathrm{SE}, \mathrm{n}=10)$

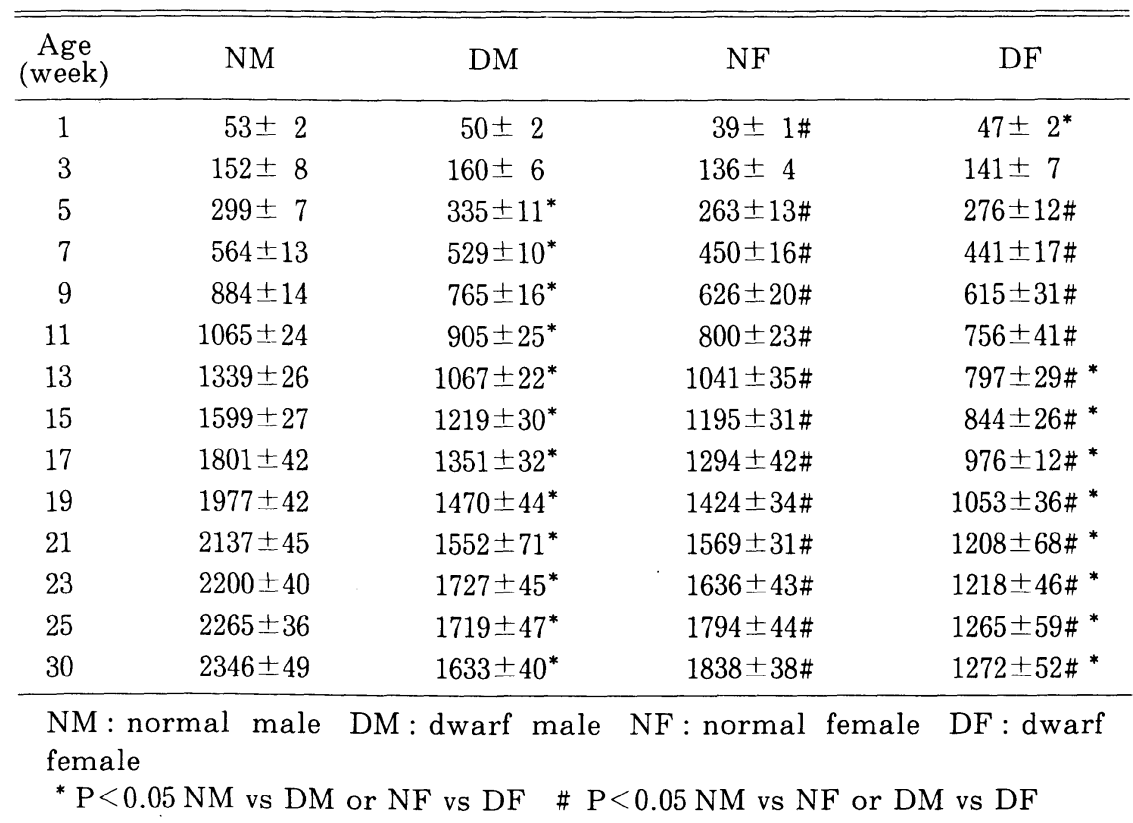


However, this difference observed between normal and dwarf male chickens was not evident in female chickens. Differences in erythrocyte counts between sexes were also significant in both lines : numbers of erythrocyte in males were greater than in females throughout the growing period examined (Table 3).

Changes in hematocrit and $\mathrm{Hb}$ content with increasing age were comparable to those in erythrocyte count (Tables 4 and 5). This is consistent with the fact that hematocrit is largely composed of erythrocytes and $\mathrm{Hb}$ contents parallel to erythrocyte

Table 2. Shank-toe lengths $(\mathrm{cm})($ Mean $\pm \mathrm{SE}, \mathrm{n}=10)$

\begin{tabular}{|c|c|c|c|c|}
\hline $\begin{array}{c}\text { Age } \\
\text { (week) }\end{array}$ & NM & $\mathrm{DM}$ & NF & DF \\
\hline 1 & $5.0 \pm 0.1$ & $5.0 \pm 0.1$ & $4.8 \pm 0.1$ & $4.8 \pm 0.2$ \\
\hline 3 & 7. $3 \pm 0.1$ & $6.9 \pm 0.1$ & $6.9 \pm 0.1 \#$ & $6.9 \pm 0.1$ \\
\hline 5 & $9.2 \pm 0.1$ & $8.9 \pm 0.1^{*}$ & $8.9 \pm 0.5$ & $8.7 \pm 0.4$ \\
\hline 7 & $11.8 \pm 0.1$ & $10.5 \pm 0.1^{*}$ & $10.5 \pm 0.1 \#$ & $10.0 \pm 0.1 \#$ \\
\hline 9 & $14.6 \pm 0.1$ & $12.3 \pm 0.1^{*}$ & $12.0 \pm 0.1 \#$ & $10.8 \pm 0.2 \#^{*}$ \\
\hline 11 & $14.9 \pm 0.1$ & $12.3 \pm 0.1^{*}$ & $12.9 \pm 0.1 \#$ & $10.7 \pm 0.5 \# *$ \\
\hline 13 & $16.3 \pm 0.1$ & $12.2 \pm 0.1^{*}$ & $12.8 \pm 0.2 \#$ & $10.3 \pm 0.1 \#^{*}$ \\
\hline 15 & $17.0 \pm 0.2$ & $12.5 \pm 0.1^{*}$ & $13.8 \pm 0.1 \#$ & $10.5 \pm 0.1 \#^{*}$ \\
\hline 17 & $17.6 \pm 0.2$ & $12.9 \pm 0.1^{*}$ & $14.5 \pm 0.2 \#$ & $10.5 \pm 0.1 \#^{*}$ \\
\hline 19 & $17.3 \pm 0.1$ & $12.3 \pm 0.1^{*}$ & $14.1 \pm 0.2 \#$ & $10.6 \pm 0.1 \#^{*}$ \\
\hline 21 & $17.5 \pm 0.2$ & $12.9 \pm 0.2^{*}$ & $14.5 \pm 0.2 \#$ & $11.1 \pm 0.2 \#^{*}$ \\
\hline 23 & 17. $2 \pm 0.1$ & $13.2 \pm 0.1^{*}$ & $14.1 \pm 0.2 \#$ & $10.9 \pm 0.2 \#^{*}$ \\
\hline 25 & $17.8 \pm 0.2$ & $13.1 \pm 0.1^{*}$ & $14.7 \pm 0.2 \#$ & $11.2 \pm 0.1 \#^{*}$ \\
\hline 30 & $17.3 \pm 0.2$ & $13.3 \pm 0.1^{*}$ & $14.5 \pm 0.1 \#$ & $10.8 \pm 0.1 \#^{*}$ \\
\hline
\end{tabular}

NM, DM, NF, DF, ${ }^{*}, \#:$ See Table 1 !

Table 3. Erythrocyte counts $\left(\times 10^{6} / \mathrm{mm}^{3}\right)($ Mean $\pm \mathrm{SE},(\mathrm{n}))$

\begin{tabular}{cclll}
\hline \hline $\begin{array}{c}\text { Age } \\
\text { (week) }\end{array}$ & \multicolumn{1}{c}{$\mathrm{NM}$} & \multicolumn{1}{c}{$\mathrm{DM}$} & \multicolumn{1}{c}{$\mathrm{NF}$} & \multicolumn{1}{c}{$\mathrm{DF}$} \\
\hline 1 & $2.30 \pm 0.06(6)$ & $2.16 \pm 0.03(7)^{*}$ & $2.17 \pm 0.03(10)$ & $2.00 \pm 0.06(8)$ \\
3 & $2.33 \pm 0.05(9)$ & $2.29 \pm 0.05(8)$ & $2.38 \pm 0.08(5)$ & $2.14 \pm 0.11(7)$ \\
5 & $2.39 \pm 0.02(10)$ & $2.34 \pm 0.02(10)$ & $2.24 \pm 0.06(10) \#$ & $2.23 \pm 0.07(10)$ \\
7 & $2.39 \pm 0.02(10)$ & $2.49 \pm 0.04(10)^{*}$ & $2.21 \pm 0.10(7)$ & $2.24 \pm 0.07(8)$ \\
9 & $2.57 \pm 0.19(10)$ & $2.73 \pm 0.19(10)^{*}$ & $2.23 \pm 0.05(8) \#$ & $2.27 \pm 0.04(8) \#$ \\
11 & $2.49 \pm 0.03(10)$ & $2.67 \pm 0.02(10)^{*}$ & $2.20 \pm 0.08(9) \#$ & $2.39 \pm 0.08(8) \#$ \\
13 & $2.45 \pm 0.03(10)$ & $2.62 \pm 0.03(10)^{*}$ & $2.41 \pm 0.04(10)$ & $2.42 \pm 0.05(10) \#$ \\
15 & $2.61 \pm 0.03(10)$ & $2.74 \pm 0.03(10)^{*}$ & $2.63 \pm 0.08(10)$ & $2.69 \pm 0.05(10)$ \\
17 & $2.78 \pm 0.03(10)$ & $3.06 \pm 0.04(10)^{*}$ & $2.48 \pm 0.04(10) \#$ & $2.73 \pm 0.05(10) \#{ }^{*}$ \\
19 & $2.82 \pm 0.04(10)$ & $3.20 \pm 0.05(10)^{*}$ & $2.29 \pm 0.05(10) \#$ & $2.47 \pm 0.23(10) \#$ \\
21 & $2.81 \pm 0.03(10)$ & $3.24 \pm 0.05(10)^{*}$ & $2.53 \pm 0.04(10) \#$ & $2.62 \pm 0.04(10) \#$ \\
23 & $2.88 \pm 0.04(10)$ & $3.26 \pm 0.03(10)^{*}$ & $2.42 \pm 0.04(10) \#$ & $2.57 \pm 0.05(10) \#{ }^{*}$ \\
25 & $3.11 \pm 0.04(10)$ & $3.37 \pm 0.02(10)^{*}$ & $2.38 \pm 0.08(10) \#$ & $2.38 \pm 0.05(10) \#$ \\
30 & $3.68 \pm 0.06(10)$ & $3.94 \pm 0.04(10)^{*}$ & $2.34 \pm 0.04(10) \#$ & $2.31 \pm 0.08(10) \#$ \\
\hline
\end{tabular}

NM, DM, NF, DF, ${ }^{*}, \#$ : See Table 1! 
Table 4. Hematocrits (\%) (Mean \pm SE, (n))

\begin{tabular}{cllll}
\hline $\begin{array}{c}\text { Age } \\
\text { (week) }\end{array}$ & \multicolumn{1}{c}{ NM } & \multicolumn{1}{c}{ DM } & \multicolumn{1}{c}{$\mathrm{NF}$} & \multicolumn{1}{c}{ DF } \\
\hline 1 & $31.5 \pm 0.9(6)$ & $30.6 \pm 0.5(7)$ & $33.6 \pm 0.5(10)$ & $30.4 \pm 0.6(8)^{*}$ \\
3 & $30.7 \pm 0.8(9)$ & $30.4 \pm 0.8(8)$ & $33.1 \pm 0.7(7) \#$ & $29.3 \pm 1.5(7)$ \\
5 & $30.9 \pm 0.4(10)$ & $30.7 \pm 0.7(10)$ & $31.5 \pm 0.4(10)$ & $30.8 \pm 1.0(8)$ \\
7 & $29.1 \pm 0.5(10)$ & $30.1 \pm 0.5(10)$ & $28.9 \pm 0.6(8)$ & $28.3 \pm 0.5(8) \#$ \\
9 & $30.5 \pm 0.4(10)$ & $33.3 \pm 0.7(10)^{*}$ & $30.3 \pm 0.5(8)$ & $31.1 \pm 0.9(8)$ \\
11 & $29.5 \pm 0.6(10)$ & $32.0 \pm 0.5(10)^{*}$ & $29.8 \pm 0.4(9)$ & $31.8 \pm 0.5(8)$ \\
13 & $29.3 \pm 0.5(10)$ & $32.5 \pm 0.8(10)^{*}$ & $32.3 \pm 0.5(10) \#$ & $33.2 \pm 0.7(10)$ \\
15 & $32.4 \pm 0.7(10)$ & $36.0 \pm 0.7(10)^{*}$ & $33.1 \pm 0.8(10)$ & $37.0 \pm 0.8(10)^{*}$ \\
17 & $31.4 \pm 0.5(10)$ & $39.3 \pm 0.8(10)^{*}$ & $33.1 \pm 0.5(10) \#$ & $36.9 \pm 0.9(10)^{*}$ \\
19 & $35.3 \pm 0.7(10)$ & $44.7 \pm 1.2(10)^{*}$ & $31.8 \pm 1.5(10) \#$ & $34.7 \pm 1.4(10)^{*} \#$ \\
21 & $36.3 \pm 1.1(10)$ & $44.6 \pm 0.9(10)^{*}$ & $30.6 \pm 0.6(10) \#$ & $33.4 \pm 0.5(10)^{*} \#$ \\
23 & $39.6 \pm 0.7(10)$ & $46.3 \pm 0.5(10)^{*}$ & $31.2 \pm 0.9(10) \#$ & $32.0 \pm 1.0(10) \#$ \\
25 & $42.5 \pm 1.5(10)$ & $48.7 \pm 1.0(10)^{*}$ & $31.4 \pm 0.3(10) \#$ & $31.0 \pm 0.6(10) \#$ \\
30 & $41.6 \pm 0.9(10)$ & $50.0 \pm 0.9(10)^{*}$ & $30.0 \pm 0.5(10) \#$ & $29.8 \pm 0.7(10) \#$ \\
\hline
\end{tabular}

NM, DM, NF, DF, ${ }^{*}$, : See Table 1 !

Table 5. Hemoglobin concentrations (g/100 ml) (Mean $\pm \mathrm{SE},(\mathrm{n}))$

\begin{tabular}{ccccc}
\hline \hline $\begin{array}{c}\text { Age } \\
\text { (week) }\end{array}$ & NM & \multicolumn{1}{c}{ DM } & \multicolumn{1}{c}{ NF } & DF \\
\hline 1 & $8.60 \pm 0.28(6)$ & $7.75 \pm 0.19(7)^{*}$ & $8.12 \pm 0.16(10)$ & $7.22 \pm 0.21(8)^{*}$ \\
3 & $8.41 \pm 0.18(9)$ & $8.16 \pm 0.18(8)$ & $8.62 \pm 0.37(7)$ & $7.30 \pm 0.27(7)^{*} \#$ \\
5 & $7.86 \pm 0.06(10)$ & $7.76 \pm 0.16(10)$ & $8.57 \pm 0.17(10) \#$ & $7.65 \pm 0.16(8)^{*}$ \\
7 & $7.59 \pm 0.16(10)$ & $7.87 \pm 0.15(10)$ & $8.05 \pm 0.22(8)$ & $8.07 \pm 0.16(8)$ \\
9 & $7.67 \pm 0.15(10)$ & $8.76 \pm 0.19(10)^{*}$ & $8.40 \pm 0.25(8) \#$ & $8.64 \pm 0.28(8)$ \\
11 & $8.21 \pm 0.17(10)$ & $8.95 \pm 0.19(10)^{*}$ & $8.39 \pm 0.19(9)$ & $9.00 \pm 0.23(8)$ \\
13 & $7.63 \pm 0.17(10)$ & $8.51 \pm 0.26(10)^{*}$ & $8.89 \pm 0.19(10) \#$ & $9.38 \pm 0.22(10) \#$ \\
15 & $8.48 \pm 0.13(10)$ & $9.44 \pm 0.24(10)^{*}$ & $9.65 \pm 0.03(10) \#$ & $11.13 \pm 0.30(10)^{*} \#$ \\
17 & $8.90 \pm 0.18(10)$ & $10.59 \pm 0.30(10)^{*}$ & $9.13 \pm 0.23(10)$ & $10.30 \pm 0.28(10)^{*}$ \\
19 & $10.40 \pm 0.21(10)$ & $12.76 \pm 0.31(10)^{*}$ & $8.52 \pm 0.51(10) \#$ & $9.84 \pm 0.71(10)^{*} \#$ \\
21 & $10.62 \pm 0.31(10)$ & $12.95 \pm 0.24(10)^{*}$ & $8.83 \pm 0.20(10) \#$ & $9.85 \pm 0.28(10)^{*} \#$ \\
23 & $11.02 \pm 0.27(10)$ & $12.41 \pm 0.29(10)^{*}$ & $8.40 \pm 0.34(10) \#$ & $8.82 \pm 0.36(10) \#$ \\
25 & $12.49 \pm 0.20(10)$ & $13.32 \pm 0.23(10)^{*}$ & $8.62 \pm 0.11(10) \#$ & $8.50 \pm 0.15(10) \#$ \\
30 & $12.78 \pm 0.27(10)$ & $15.19 \pm 0.26(10)^{*}$ & $8.12 \pm 0.09(10) \#$ & $8.07 \pm 0.12(10) \#$ \\
\hline
\end{tabular}

NM, DM, NF, DF, ${ }^{*}$, \# : See Table 1!

counts.

The present results on hematological examination in dwarf chickens are in good agreement with previous reports ${ }^{6,7)}$ on $d w$ chickens where $d w$ gene had been incorporated into White Leghorn or a meat stock, and blood samples were taken at 25 and 40 or 68 weeks of age. Accordingly, it is likely that dw gene increases erythrocyte count and thereby hematocrit and $\mathrm{Hb}$ when the dwarfing effect on skeletal growth became apparent, irrespective of types of chickens to which the gene was transferred. 
This appears to be paradoxical, because the dwarf chicken having a smaller sized body will require less oxygen than the corresponding normal chicken.

The increased number of erythrocyte in dwarfs having low circulating IGF-1 levels also contradict to the findings in mammls ${ }^{2,3)}$ in which there is a positive relation between IGF-1 concentrations and erythrocyte production. However, it is possible that a higher $\mathrm{GH}$ level ${ }^{10)}$ itself stimulates erythropoiesis in the dwarf chicken. In fact, our recent study (WAKITA et al. unpublished) revealed that hypophysectomy reduces erythrocyte count, whereas purified chicken GH injection to hypophysectomized chickens restores erythrocyte to sham control level without the restoration of IGF-1 level in circulation. This seems to support our presumption descrbied above : the stimulative effect of $\mathrm{GH}$ on erythropoisis in dwarf chickens, though more studies are clearly required.

\section{References}

1) Decuypere, E., L.M. Huybrechts, E.R. Kühn, M. Tixier-Boichard and P. Merat (1991) Physiological alterations associated with the chicken sex-linked dwarfing gene Crit. Rev. Poultry Biol. 3 : 191-221.

2) Kurtz, A., J. Zapf, K.-U. Eckardt, G. Clemons, E.R. Froesch and C. Bauer (1988) Insulin-like growth factor 1 stimulates erythropoiesis in hypophysectomized rats. Proc. Natl. Acad. Sci. 85 : 7825-7829.

3) Kurtz, A., R. Matter, K.-U. Eckardt and J. ZapF (1990) Erythropoiesis, serum erythropoietin, and serum IGF-1 in rats during accelerated growth. Acta Endocrinol (Copenh) 122: 323-328.

4) Hoshino, S., M. Wakita, M. Suzuki and K. Yamamoto (1982) Changes in a somatomedin-like factor and immunoassayable growth hormone during growth of normal and dwarf pullets and cockerels. Poultry Sci. 61 : 777-784.

5) Huybrechts, L.M., D.B. King, T.J. Lauterio, J.A. Marsh and C.G. Scanes (1985) Plasma concentrations of somatomedin-C in hypophysectomized, dwarf and intact growing domestic fowl as determined by heterologous radioimmunoassay. J. Endocrinol. 104 : 233-236.

6) Reddy, P.R.K., W.B. Gross, H.P. Van Krey and P.B. Siegel (1975) Blood parameters of dwarf and normal pullets from growth selected lines before and after Escherichia coli challenge. Poultry Sci. 54:674-681.

7) Wood, A.S., B.S. Reinhart, G. Rajaratnam and J.D. Summers (1971) A comparison of the blood constituents of dwarf versus non-dwarf birds. Poultry Sci. $50: 804-807$.

8) Watanabe, K., H. Hayakawa, T. Takai, S. Ebisawa and T. Tsuji (1978) On the peformance of a dwarf Rhodehorn. Res. Report Gifu Poult. Breeding Station 25 : $10-16$.

9) Hoshino, S., S. Matsuzaki, M. Wakita, Y. Kobayashi and M. Suzuki (1989) Cartilage response to thyroid hormones in the sex-linked dwarf chicken. Comp. Biochem. Physiol. 93 A : 583-585.

10) Hoshino, S., L.M. Huybrechts, M. Wakita, Y. Kobayashi I. Yamamoto, Y. Nishiguchi, V.M. DARRAS, E. DeCuYpere and E.R. KüHN (1990) Influence of estradiol-17 on plasma thyroid hormone response to thyrotropin releasing hormone in chick embryos and pullets. Jpn. Poult. Sci. 27 : 291-297. 